\title{
Intermittent selective clamping improves rat liver regeneration by attenuating oxidative and endoplasmic reticulum stress
}

\author{
I Ben Mosbah ${ }^{1,2}$, H Duval ${ }^{\dagger, 1,2}$, S-F Mbatchi ${ }^{1,2}$, C Ribault ${ }^{1,2}$, S Grandadam ${ }^{1,2,3}$, J Pajaud ${ }^{1,2}$, F Morel $^{1,2}$, K Boudjema $^{1,2,3}$, \\ P Compagnon ${ }^{\ddagger 1,2,3,4}$ and A Corlu ${ }^{\star, 1,2,4}$
}

Intermittent clamping of the portal trial is an effective method to avoid excessive blood loss during hepatic resection, but this procedure may cause ischemic damage to liver. Intermittent selective clamping of the lobes to be resected may represent a good alternative as it exposes the remnant liver only to the reperfusion stress. We compared the effect of intermittent total or selective clamping on hepatocellular injury and liver regeneration. Entire hepatic lobes or only lobes to be resected were subjected twice to $10 \mathrm{~min}$ of ischemia followed by $5 \mathrm{~min}$ of reperfusion before hepatectomy. We provided evidence that the effect of intermittent clamping can be damaging or beneficial depending to its mode of application. Although transaminase levels were similar in all groups, intermittent total clamping impaired liver regeneration and increased apoptosis. In contrast, intermittent selective clamping improved liver protein secretion and hepatocyte proliferation when compared with standard hepatectomy. This beneficial effect was linked to better adenosine-5'-triphosphate (ATP) recovery, nitric oxide production, antioxidant activities and endoplasmic reticulum adaptation leading to limit mitochondrial damage and apoptosis. Interestingly, transient and early chaperone inductions resulted in a controlled activation of the unfolded protein response concomitantly to endothelial nitric oxide synthase, extracellular signal-regulated kinase-1/2 (ERK1/2) and p38 MAPK activation that favors liver regeneration. Endoplasmic reticulum stress is a central target through which intermittent selective clamping exerts its cytoprotective effect and improves liver regeneration. This procedure could be applied as a powerful protective modality in the field of living donor liver transplantation and liver surgery.

Cell Death and Disease (2014) 5, e1107; doi:10.1038/cddis.2014.65; published online 6 March 2014

Subject Category: Experimental Medicine

Total inflow occlusion through clamping of the portal triad has been shown to minimize blood loss and transfusion during liver surgery. ${ }^{1}$ The main drawback of this procedure is ischemic injury to liver parenchyma that increases postoperative morbidity and mortality. ${ }^{2,3}$ Intermittent occlusion of the hepatic inflow pedicle with intervals of reperfusion reduces the consequences of splanchnic congestion and therefore decreases hepatic ischemia/reperfusion (I/R) injury. ${ }^{2,4}$ Ischemia decreases the delivery of oxygen and substrates to a level inadequate to maintain cellular energy. This phenomenon provokes a rapid and severe decline in high-energy phosphate compound levels, generalized cell membrane depolarization as well as a large increase in intracellular $\mathrm{Ca}^{2+}$ concentration accompanied by $\mathrm{Ca}^{2+}$ depletion from the endoplasmic reticulum (ER). This mobilization of $\mathrm{Ca}^{2+}$ stores from ER plays a significant role in modulating the mitochondrial $\mathrm{Ca}^{2+}$ responses and the susceptibility of hepatocytes to apoptotic signals. ${ }^{5,6}$

\footnotetext{
${ }^{1}$ Inserm, UMR 991, Liver, Metabolisms and Cancer, CHU Pontchaillou, F-35033 Rennes, France; ${ }^{2}$ Université de Rennes 1, F-35043 Rennes, France and ${ }^{3}$ Service de Chirurgie Hépatobiliaire et Digestive, CHU Pontchaillou, Rennes, France

*Corresponding author: A Corlu, UMR 991, Foie, Métabolismes et Cancer, CHU Pontchaillou, Rennes 35033, France. Tel: +33 2323 3870; Fax: +33 2 9954 0137; E-mail: anne.corlu@inserm.fr

${ }^{4}$ These authors contributed equally to this work.

'Current address: Equipe EG2B, Laboratoire LIMATB, Université de Bretagne Sud, F-56017 Vannes, France

${ }^{\ddagger}$ Current address: Service de Chirurgie Digestive et Hépatobiliaire-Transplantation Hépatique, Hôpital Henri Mondor, Assistance Publique-Hôpitaux de Paris, Créteil, France; IMRB-INSERM U955, équipe 21, Université Paris-Est Créteil, Créteil, France

Keywords: oxidative and endoplasmic reticulum stress; ischemia reperfusion injury; unfolded protein response; mitochondrial damage

Abbreviations: I/R, ischemia/reperfusion; $E R$, endoplasmic reticulum; ROS, reactive oxygen species; UPR, unfolded protein response; IRE1, inositol-requiring enzyme 1; PERK, PKR-like ER kinase; ATF6, activating transcription factor 6; GRP78, 78-kDa glucose-regulated/binding immunoglobulin protein; elF2 $\alpha$, eukaryotic translation initiation factor $2 \alpha$; JNK, C-Jun N-terminal kinase; CHOP, C/EBP-homologous protein; NO, nitric oxide; ITC, intermittent total clamping; ISC, intermittent selective clamping; ATP, adenosine-5'-triphosphate; PCNA, proliferating cell nuclear antigen; PH, standard hepatectomy; RT-PCR, real-time PCR; NCPH, hepatectomy after liver mobilization without clamping; TCPH, hepatectomy after intermittent total clamping; RPH, hepatectomy after intermittent selective clamping; ALT, alanine aminotransferase; AST, aspartate aminotransferase; LDH, lactate dehydrogenase; MDA, malondialdehyde; SOD, superoxide dismutase; GADD34, growth arrest and DNA damage gene-34; EDEM, ER degradation-enhancing $\alpha$-mannosidase-like protein; ASNS, aspargine synthetase; GRP94, 94-kDa glucose-regulated/binding immunoglobulin protein; PVDF, polyvinylidene fluoride; TRAF2, tumor necrosis factor-associated factor 2; XBP-1(S), spliced X-box-binding protein-1; ERK, extracellular signal-regulated kinase; p38, mitogen-activated protein kinase; eNOS, endothelial nitric oxide synthase; iNOS, inducible nitric oxide synthase; TUNEL, terminal deoxynucleotidyl transferase-mediated dUTP biotin nick end labeling; BrdU, 5-bromo-2'deoxyuridine

Received 28.10.13; revised 10.1.14; accepted 27.1.14; Edited by M Federici
} 
Consequently, although restoration of oxygen during the reperfusion period has an important benefit and activates some events that normally occur after hepatectomy, reactive oxygen species (ROS) and cytokines highly produced during ischemia lead to additional cell injury and trigger the apoptotic machinery in hepatocytes. ${ }^{7}$

In addition to functions that are strictly involved in protein folding, trafficking, secretion and degradation, the ER is now recognized to have important physiological roles in metabolism, inflammation as well as cell differentiation and survival. ${ }^{8-11}$ Therefore, ER stress that results from an imbalance between the cellular demand for ER function and ER capacity emerges as an important player in inflammatory responses and activation of cell death mechanisms in liver during I/R. 8,12,13 Indeed, vascular occlusion affects the ability of ER to synthesize, fold and sort proteins and thus leads to ER stress. ${ }^{5,12,13}$ In response to ER stress, a signal transduction cascade termed unfolded protein response (UPR) is induced to upregulate the expression of ER chaperones, to inhibit the protein synthesis and to activate protein degradation. ${ }^{8,12}$ Three ER transmembrane proteins are identified as signal sensors of ER stress: inositol-requiring enzyme 1 (IRE1), PKR-like ER kinase (PERK) and activating transcription factor (ATF6). ${ }^{14,15}$ These proteins that are normally held in an inactive state by binding to ER chaperones such as GRP78 (78-kDa glucose-regulated/binding immunoglobulin protein)/BiP are released following ER stress. Activated PERK phosphorylates the $\alpha$-subunit of eukaryotic translation initiation factor 2 (elF2 $\alpha$ ) that results in the inhibition of protein translation and reduction of newly synthesized proteins in the ER lumen. Activated IRE1 induces upregulation of ER chaperone transcription and initiates proapoptotic responses mediated by c-JUN N-terminal kinase (JNK-1) to eliminate damaged cells. ${ }^{16,17}$ ATF6, a transcription factor released from the ER membrane, also allows transcription of genes including chaperones and foldases. However, when injury is excessive and ER function unrestored, the ER stress signal transduction pathways induce cell death through induction of transcription factor C/EBP-homologous protein $(\mathrm{CHOP}) .{ }^{15,18}$ We have previously reported, using an original rat model of intermittent selective clamping ${ }^{19}$ of the hepatic lobes to be resected, that short intermittent reperfusion stress induces mild liver injury and accelerates the regenerative response compared with a partial hepatectomy without clamping via JNK activation. ${ }^{19}$ Although intermittent clamping is being widely used in the clinical setting, experiments evaluating underlying mechanisms of action, particularly its effect on ER function, hepatic protein synthesis, energetic metabolism restoration, nitric oxide (NO) production and antioxidant activities after hepatectomy, are lacking. Here, we compare the effects of intermittent total (ITC) or selective (ISC) clamping on hepatocellular damage, the stress response and liver regeneration after partial hepatectomy. We particularly focus our attention on their potential effects on ER function during liver regeneration. We show that compared with ITC of the hepatic pedicle, ISC of the lobes to be resected not only ameliorates hepatic regeneration but also preserves liver function such as protein secretion. This better liver recovery after resection is linked to greater maintenance of antioxidant activities, enhanced preservation and restoration of tissue adenosine-5'-triphosphate (ATP) contents and better ER adaptation to $1 / \mathrm{R}$ stress, leading to limited mitochondrial damage and apoptosis.

\section{Results}

ISC improves hepatocyte proliferation and liver function. Liver regeneration following either an intermittent selective clamping of the lobes to be resected prior hepatectomy, subjecting the remnant liver only to reperfusion stress (RPH), or an intermittent clamping of the entire hepatic pedicle (TCPH) before resection, subjecting the whole liver to I/R stress, was first evaluated. Both surgical procedures were compared with standard partial hepatectomy $(\mathrm{PH})$ or lobe exteriorization and mobilization without clamping followed (NCPH) or not (SHAM) by a resection (Figure 1).

Quantification of the mitotic index, percentage of hepatocyte 5-bromo-2'deoxyuridine (BrdU) incorporation and proliferating cell nuclear antigen (PCNA) expression at different times after resection showed that liver regeneration was impaired in the TCPH group (Figures 2a-d). Mitotic index was reduced $24 \mathrm{~h}$ after hepatectomy in the TCPH group as compared with those observed in the $\mathrm{PH}$ group (Figure 2a). In addition, percentages of replicating hepatocytes were significantly lowered 21 and $24 \mathrm{~h}$ after hepatectomy in the $\mathrm{TCPH}$ group as compared with the $\mathrm{PH}$ group (Figure 2b). Similarly, PCNA protein levels were remarkably lower at 21, 24 and $48 \mathrm{~h}$ after hepatectomy in the TCPH group as compared with the PH group (Figures 2c and d). In contrast, ISC and liver mobilization before hepatectomy significantly accelerated and improved hepatocyte DNA synthesis as compared with $\mathrm{PH}$ (Figures $2 b-d$ ). PCNA expression was detected as soon as $18 \mathrm{~h}$ post hepatectomy. The mitotic index also indicated that hepatocyte division similarly arose $24 \mathrm{~h}$ after hepatectomy in both RPH and NCPH groups, whereas it occurred only at $30 \mathrm{~h}$ in $\mathrm{PH}$ (Figure 2a).

This hepatocyte proliferation ability was correlated with tissue ATP content (Figure 2e). In the NCPH group, and more pronouncedly in the RPH group, higher amount of ATP levels was detected as compared with the $\mathrm{PH}$ group at 3 and $24 \mathrm{~h}$ after hepatectomy. In contrast, reduced ATP contents were observed in the TCPH group in comparison with levels observed in the $\mathrm{PH}$ group $3 \mathrm{~h}$ after hepatectomy or in comparison with those detected in the RPH and NCPH groups at 3 and $24 \mathrm{~h}$ post hepatectomy (Figure $2 \mathrm{e}$ ).

Regarding hepatocellular injury, alanine aminotransferase (ALT) levels (Figure 2f) and damage score (Figure $2 \mathrm{~g}$ ) at $24 \mathrm{~h}$ after hepatectomy were comparable in $\mathrm{PH}, \mathrm{NCPH}, \mathrm{RPH}$ and $\mathrm{TCPH}$ (Figures $2 \mathrm{f}$ and $\mathrm{g}$ ). The aspartate aminotransferase (AST) release had similar profile to ALT (data not shown). In contrast, higher level of lactate dehydrogenase (LDH) was detected in the TCPH group when compared with other tested groups (Figure 2h).

ISC maintains antioxidant activities preventing damaging lipid peroxidation. Lipid peroxidation was used as an indirect marker of ROS-induced hepatic damage. The malondialdehyde (MDA) levels were significantly increased after hepatectomy when compared with SHAM (Figure 2i). In the TCPH group, these values were greatly increased 

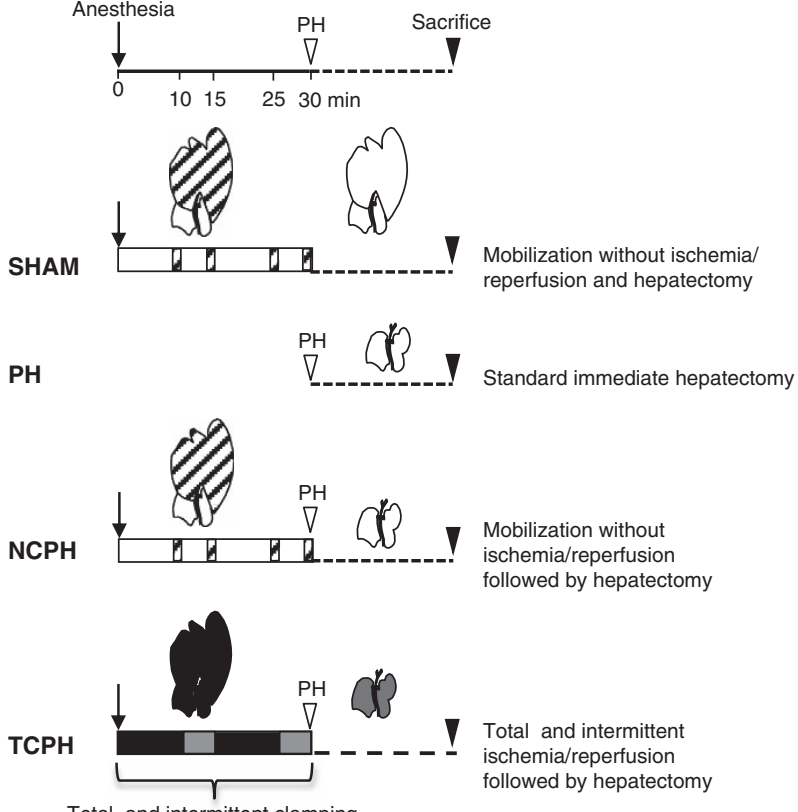

Total and intermittent clamping

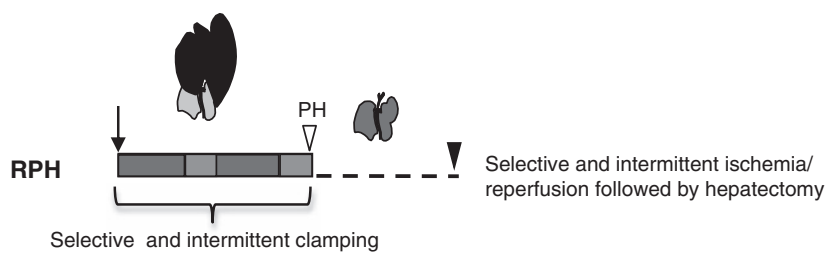

Figure 1 Schematic description of experimental groups. Animals from the SHAM group were prepared as a nonregenerative control: $70 \%$ of liver lobes were mobilized at the indicated times (dashed bars) to simulate the clamping and reperfusion procedures. In the $\mathrm{PH}$ group, the standard hepatectomy was immediately performed without mobilization. In NCPH, the same protocol as SHAM was followed by hepatectomy. In TCPH, intermittent clamping applied to entire hepatic pedicle was induced by $10 \mathrm{~min}$ of ischemia followed by $5 \mathrm{~min}$ of reperfusion two times before hepatectomy. In RPH, the same protocol as TCPH, but intermittent clamping was applied only to lobes to be resected. White bar represents no procedure on remaining lobes whereas lobes to be resected were exteriorized. Black bar represents total ischemic period and dark gray bar represents selective ischemic period on exteriorized lobes. Light gray bar corresponds to the reperfusion period

compared with the $\mathrm{PH}$ group at all time points studied and reached a peak value at $24 \mathrm{~h}$. In $\mathrm{RPH}, \mathrm{PH}$ and $\mathrm{NCPH}$ groups, MDA levels were similar $3 \mathrm{~h}$ after hepatectomy (Figure $2 \mathrm{i}$ ). However, the MDA induction at $24 \mathrm{~h}$ was limited in the NCPH as compared with the $\mathrm{PH}$ group. Notably, lesser MDA levels with no peak were observed $24 \mathrm{~h}$ after hepatectomy in the $\mathrm{RPH}$ group as compared with the $\mathrm{PH}$ and NCPH groups (Figure 2i).

In parallel, we observed a drastic reduction of antioxidant activities of superoxide dismutase (SOD) and catalase at all time points following hepatectomy, the lowest levels being observed in $\mathrm{TCPH}$ (Figures 2j and k). Interestingly, better maintenance of antioxidant activities was detected in RPH (Figures 2j and k).

ISC attenuates ER stress activation. We analyzed the mRNA gene expression and the protein levels of GRP78 and GRP94 (94-kDa glucose-regulated/binding immunoglobulin protein), two ER chaperones, and $\mathrm{CHOP}$, an effector of ER stress involved in apoptosis and DNA damage. GRP78, GRP94 and CHOP mRNA and protein levels were higher in the $\mathrm{PH}$ group relative to those observed in SHAM group (Figure 3). Both chaperones were similarly detected in the $\mathrm{PH}$ and $\mathrm{TCPH}$ groups at $3 \mathrm{~h}$ after hepatectomy (Figures $3 \mathrm{a}$, $b, d, e, g$ and $h$ ). At this time point, the mRNA and protein levels of GRP78 and GRP94 were significantly increased in the NCPH group and particularly in the RPH group when compared with those in the $\mathrm{PH}$ and TCPH groups. However, at $24 \mathrm{~h}$ post hepatectomy, their amounts significantly increased in the $\mathrm{PH}$ and TCPH groups, whereas the mRNA and protein levels remained stable or decreased in the NCPH and RPH groups, respectively (Figures $3 a, b, d, e, g$ and $h$ ). Importantly, the mRNA and protein levels of $\mathrm{CHOP}$ were greater in the TCPH group than in the $\mathrm{PH}$ group at 3 and $24 \mathrm{~h}$ post hepatectomy, whereas its amount was significantly lower in the NCPH group and particularly in the RPH group (Figures 3c, f and i). Immunolocalization of GRP78 and CHOP (Figures $4 a-d$ ) revealed that activation of ER stress occurred mainly in hepatocytes and confirmed the results obtained by real-time PCR (RT-PCR) and western blot (Figures $3 a, c, d$ and $f$ ).

As the induction of GRP78 and GRP94 as well as CHOP expression are relevant markers of UPR activation, we further examined which of the three branches of the UPR (ATF6, PERK and IRE1) were activated after liver resection (Figures 5a-c).

ISC modulates UPR signaling. Activation of ATF6 consists in the cleavage of a $90-\mathrm{kDa}$ protein (p-90ATF6 $\alpha$ or $\mathrm{p}-90 \mathrm{ATF} 6 \beta$ ) into a $50-\mathrm{kDa}$ protein $(\mathrm{p}-50 \mathrm{ATF} 6 \alpha$ or $\mathrm{p}-50$ ATF $6 \beta$ ). Phospho-50ATF $6 \alpha$ amounts were comparable in the $\mathrm{NCPH}$ and $\mathrm{PH}$ groups at 3 and $24 \mathrm{~h}$ after hepatectomy (Figure 5a). In contrast, ITC highly increased its expression compared with other groups, whereas ISC remarkably reduced $\mathrm{p}-50$ ATF $6 \alpha$ levels. Phospho-50ATF $6 \beta$ protein levels in the NCPH, TCPH and RPH groups were identical to those in the $\mathrm{PH}$ group at 3 and $24 \mathrm{~h}$ after hepatectomy (Figure $5 \mathrm{a}$ ).

PERK pathway activation was evaluated by monitoring PERK and elF $2 \alpha$ phosphorylation and ATF4 protein expression. Liver resection induced PERK and elF2 $\alpha$ phosphorylation as well as ATF4 protein accumulation (Figure 5b). Phospho-PERK, p-elF2 $\alpha$ and ATF4 levels were similar in the NCPH and $\mathrm{PH}$ groups at 3 and $24 \mathrm{~h}$ post hepatectomy, whereas their levels were significantly increased in the TCPH group as compared with the NCPH and $\mathrm{PH}$ groups. Importantly, PERK and elF2 $\alpha$ phosphorylation was reduced and ATF4 expression was diminished in the RPH group (Figure 5b).

IRE1 pathway activation was evaluated by monitoring IRE $1 \alpha$ phosphorylation, spliced X-box-binding protein-1 (XBP-1(S)) and tumor necrosis factor-associated factor 2 (TRAF2) accumulations. As shown in Figure 5c, p-IRE1 $\alpha$, XBP-1(S) and TRAF2 protein expression increased after hepatectomy as compared with levels found in the SHAM group. Liver mobilization before hepatectomy (NCPH group) resulted in similar protein levels of $\mathrm{p}-\operatorname{IRE} 1 \alpha, \mathrm{XBP}-1(\mathrm{~S})$ and TRAF2 than in the $\mathrm{PH}$ group. ITC significantly increased p-IRE1, XBP-1(S) and TRAF2 expression levels compared 

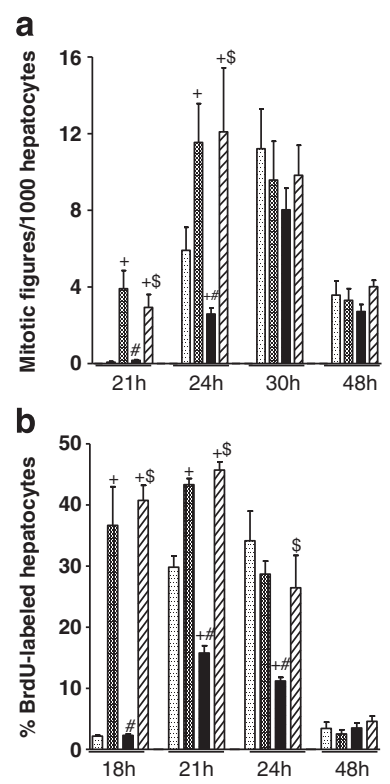

c

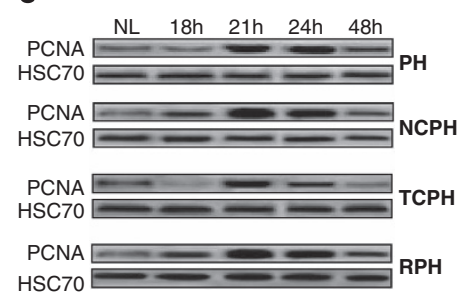

d

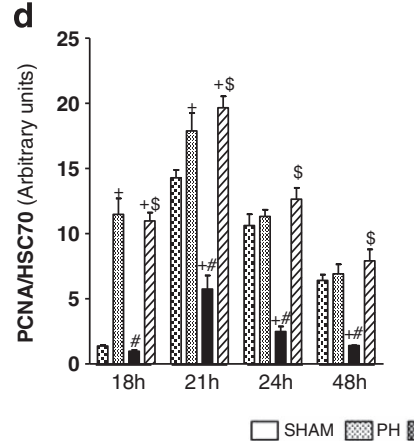

e

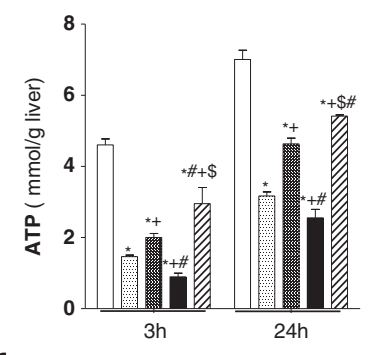

f

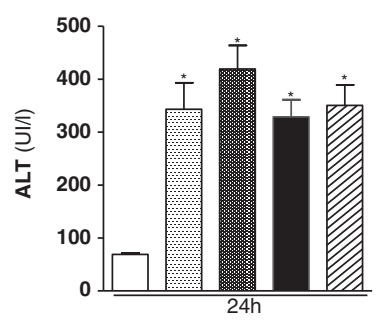

g

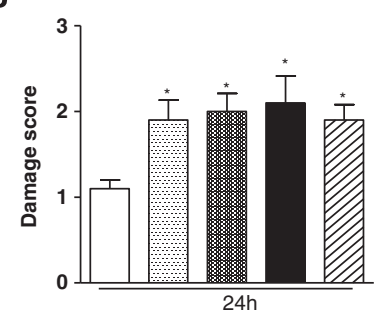

h

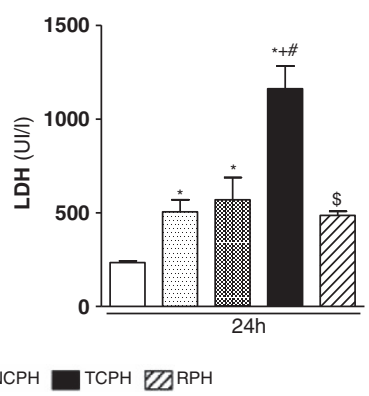

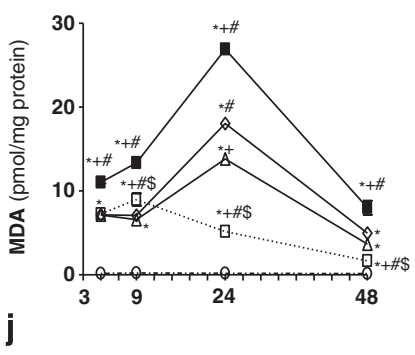

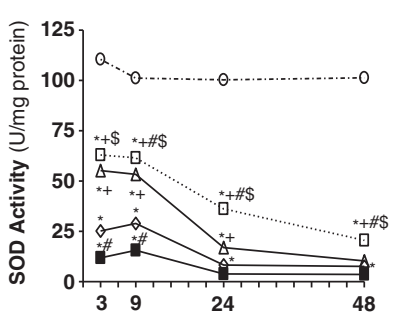

k

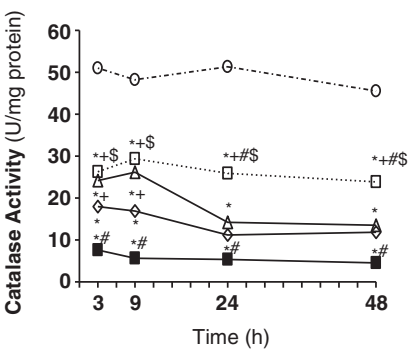

$\circ \mathrm{SHAM}-\mathrm{PH} \quad \triangle \mathrm{NCPH} \backsim \mathrm{TCPH} \square \mathrm{RPH}$

Figure 2 ISC improves hepatocytes proliferation and attenuates oxidative stress. Mitotic index (a), percentage of BrdU-positive hepatocytes (b) and PCNA protein levels (c) were measured at the indicated times. Densitometric analysis of the western blot results of PCNA (d) was performed 18, 21, 24 and $48 \mathrm{~h}$ post hepatectomy. Hepatic ATP content (e), ALT levels (f), damage score $(\mathbf{g})$ and LDH (h), hepatic MDA levels (i), superoxide dismutase (j) and catalase (k) activities were measured at the indicated times. HSC70 served as a loading control. NL, normal liver. ${ }^{*} P<0.05$ versus SHAM, ${ }^{+} P<0.05$ versus $\mathrm{PH},{ }^{\#} P<0.05$ versus NCPH and ${ }^{\$} P<0.05$ versus TCPH $(N=5)$

with those in the $\mathrm{PH}$ group. Importantly, ISC prevented these activations at 3 and $24 \mathrm{~h}$ post hepatectomy (Figure $5 \mathrm{c}$ ).

The mRNA expression analysis of ER stress target genes, GADD34 (growth arrest and DNA damage gene-34), ASNS (aspargine synthetase) and EDEM (ER degradation-enhancing $\alpha$-mannosidase-like protein), indicated that at $3 \mathrm{~h}$ post hepatectomy all these genes were less activated in $\mathrm{NCPH}$ than in the $\mathrm{PH}$ group (Figure 6a). The greatest activation of these genes at this time point was observed in the TCPH group. In contrast, GADD34, ASNS and EDEM mRNA levels was reduced in the RPH group when compared with other groups. No difference was observed for all these genes between $\mathrm{PH}, \mathrm{NCPH}, \mathrm{TCPH}$ and $\mathrm{RPH}$ groups at $24 \mathrm{~h}$ after hepatectomy (Figure 6a).

ISC induces survival signaling through MAPK activation and limits the execution of ER stress. Phosphorylation levels of JNK1/2, mitogen-activated protein kinase (p38) and extracellular signal-regulated kinase-1/2 (ERK1/2) were not affected by liver mobilization and thus were equivalent in $\mathrm{PH}$ and NCPH (Figure 6b). Interestingly, at 3 and $24 \mathrm{~h}$ after hepatectomy, ITC significantly increased $\mathrm{p}-\mathrm{JNK} 1 / 2$ and decreased $p$-p38 and $p$-ERK1/2 protein levels compared with those observed in the $\mathrm{PH}$ and $\mathrm{NCPH}$ groups. In contrast, 

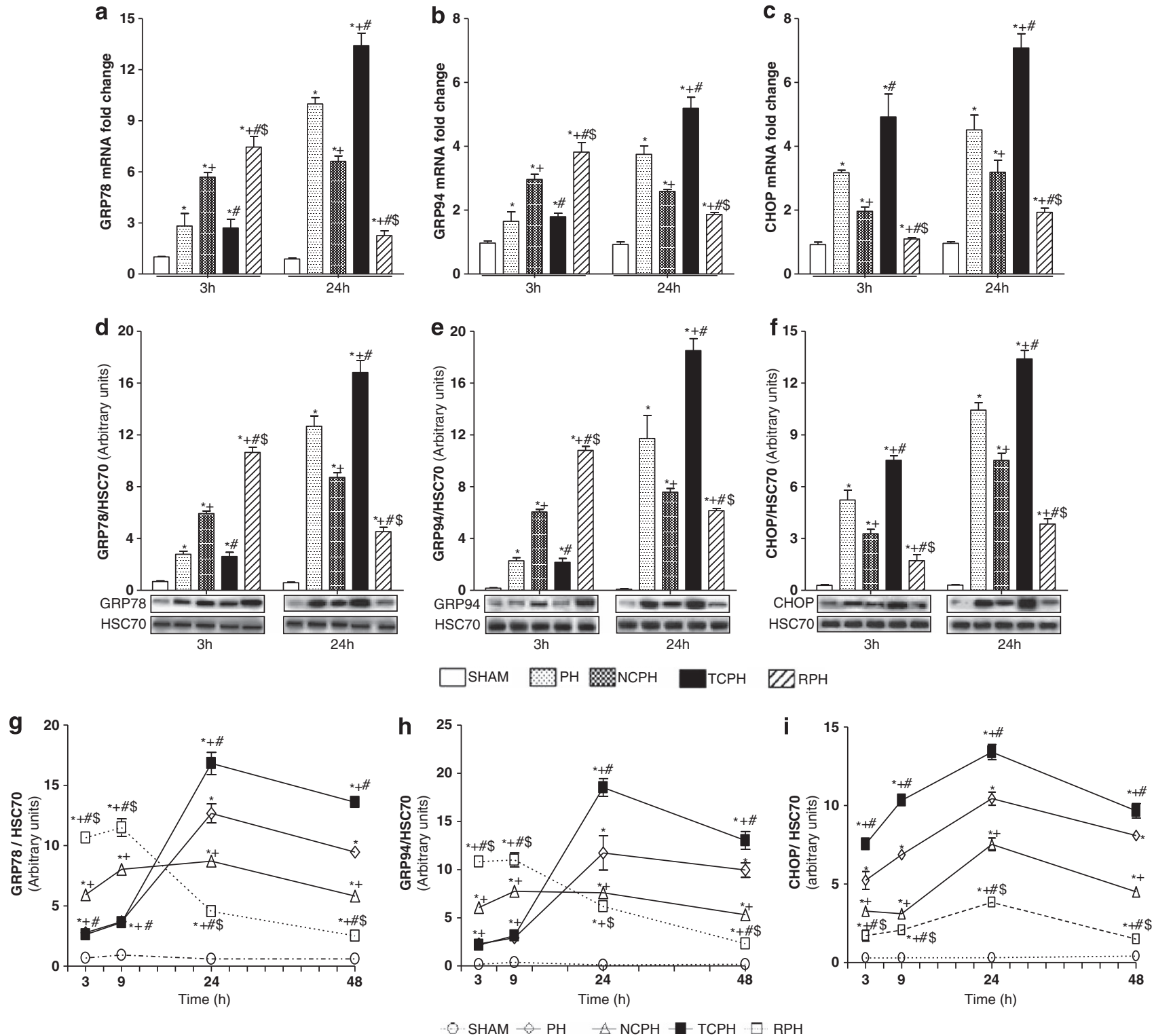

Figure 3 Time-dependent effects of ISC on ER stress markers. GRP78 (a), GRP94 (b) and CHOP (c) mRNA expressions as well as GRP78 (d), GRP94 (e) and CHOP (f) proteins levels were measured 3 and $24 \mathrm{~h}$ post hepatectomy. Densitometric analysis of the western blot results of GRP78 (g), GRP94 (h) and CHOP (i) was performed 3,9 , 24 , and 48 h post hepatectomy. ${ }^{*} P<0.05$ versus SHAM, ${ }^{+} P<0.05$ versus $\mathrm{PH},{ }^{\#} P<0.05$ versus $\mathrm{NCPH}$ and ${ }^{\$} P<0.05$ versus TCPH $(N=5)$

lower p-JNK1/2 and higher p-p38 and p-ERK1/2 levels were observed following ISC procedure compared with $\mathrm{PH}$ and NCPH (Figure 6b).

Considering the connection between ER stress, mitochondrial damage and apoptosis, we analyzed caspase 3, 9 and 12 activation, mitochondrial and cytosolic cytochrome $c$ content as well as apoptotic index by TUNEL (terminal deoxynucleotidyl transferase-mediated dUTP biotin nick end labeling) at 3 and $9 \mathrm{~h}$ after hepatectomy. Comparable levels of caspase 12, cleaved caspases 3, 9 and cytochrome $c$ (Figures $6 \mathrm{c}$ and d) as well as similar percentage of TUNEL-positive cells were observed between $\mathrm{NCPH}$ and $\mathrm{PH}$ groups (Figures $7 a$ and $b$ ). ITC significantly increased caspase 3 and 9 activation and caspase 12 induction, mitochondrial cytochrome $c$ release into cytoplasm and TUNEL staining compared with those observed in other groups (Figures $6 \mathrm{c}$ and $\mathrm{d}$ and Figures $7 \mathrm{a}$ and b). Importantly, ISC reduced apoptosis and improved mitochondrial integrity compared with the $\mathrm{PH}$ group. After hepatectomy, caspase 3 activity increased, peaking at $9 \mathrm{~h}$ and thereafter decreased with time in the $\mathrm{PH}$ and $\mathrm{NCPH}$ groups (Figure 6e). In the TCPH group, caspase 3 activity appeared as early as $3 \mathrm{~h}$ and reached higher levels compared with the $\mathrm{PH}$ group. Importantly, caspase 3 activity was significantly lower and was no longer detectable $9 \mathrm{~h}$ after ISC procedure compared with PH (Figure 6e).

ISC favors NO production. NO and ROS disturb ER functions, leading to ER stress-mediated apoptosis. NO production, which was reflected by the nitrites and nitrates contents, was significantly reduced in the TCPH group as 
a

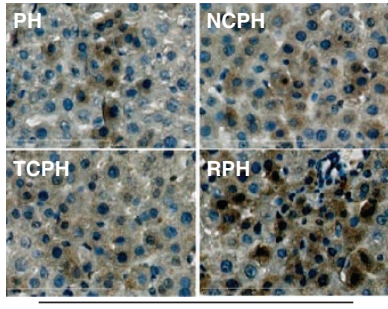

3h

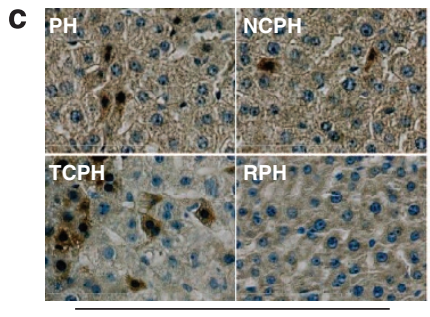

3h

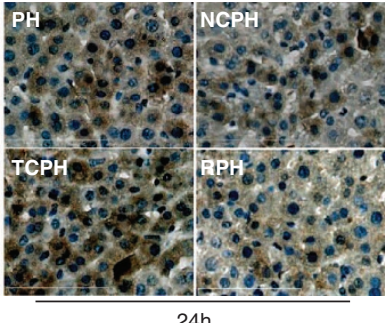

$24 \mathrm{~h}$

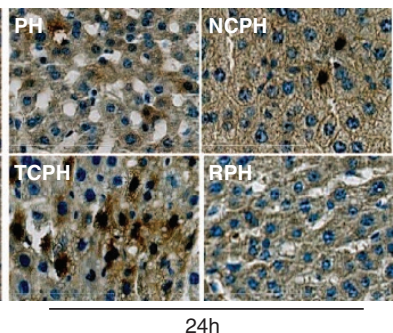

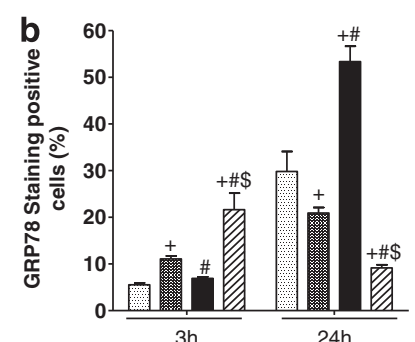

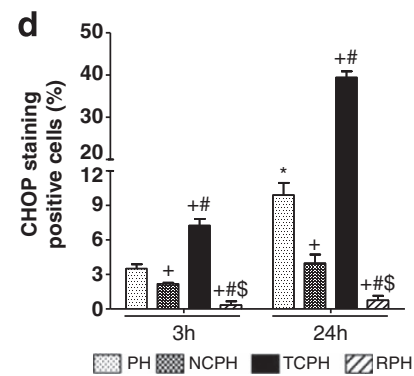

Figure 4 ISC attenuates GRP78 and CHOP expression. Immunohistochemical detection ( $\times$ 40) and percentage of GRP78-positive (a and b) and CHOP-positive (c and d) hepatocytes were analyzed 3 and $24 \mathrm{~h}$ post hepatectomy. ${ }^{+} P<0.05$ versus $\mathrm{PH},{ }^{\#} P<0.05$ versus $\mathrm{NCPH}$ and ${ }^{\$} P<0.05$ versus TCPH $(\mathrm{N}=5)$

compared with the levels observed in the $\mathrm{PH}$ and $\mathrm{NCPH}$ groups at 3 and $24 \mathrm{~h}$ after hepatectomy (Figure $7 \mathrm{c}$ ). On the contrary, this amount was significantly increased in $\mathrm{RPH}$. Importantly, we showed that newly synthesized NO originated from endothelial nitric oxide synthase (eNOS) as enhanced expression was only observed in the RPH group as compared with the $\mathrm{NCPH}$ and $\mathrm{PH}$ groups (Figure $7 \mathrm{~d}$ ). Indeed, expression of inducible nitric oxide synthase (iNOS) was barely detectable in all studied groups (data not shown).

ISC maintains liver protein secretion. To correlate the adaptive ER stress response after hepatectomy to liver protein secretion, we quantified the tissue expression of albumin and transferrin (Figures $7 e$ and $\mathrm{f}$ ). Both protein amounts were decreased in the TCPH group and increased in the RPH group as compared with the $\mathrm{PH}$ and $\mathrm{NCPH}$ groups at 3 and $24 \mathrm{~h}$ after hepatectomy (Figures $7 \mathrm{e}$ and f).

\section{Discussion}

Hepatic I/R injury still remains an unresolved problem that has immediate and deleterious effects on the outcome of patients after liver surgery. ${ }^{20}$ The pathogenesis underlying I/R injury is complex, involving different cell types and signaling mechanisms intrinsic and/or mobilized to the liver. There is substantial evidence that generation of ROS, disturbances of hepatic microcirculation, severe mitochondrial damage and alterations of the secretory pathways, particularly at the level of ER function, are involved in this syndrome.5,12,14 Currently, intermittent Pringle maneuver remains the most frequently used technique to limit $\mathrm{I} / \mathrm{R}$ injury in the absence of clinical benefit of others interventions. Here, we provide evidence that intermittent clamping can be damaging or beneficial depending to its mode of application. ISC of the lobes to be resected promoted better liver function and regeneration by favoring ATP-level restorations, NO production as well as maintenance of antioxidant and ER functions. In contrast, although no hepatic necrosis and leukocyte infiltration were observed following ITC before hepatectomy (data not shown), high levels of LDH were detected when compared with other tested groups. This increased level of LDH reflected general tissue damage that could be induced by splanchnic blood stasis during the clamping period and endotoxin release following intestinal congestion-reperfusion. ${ }^{21}$ Unexpectedly, the ITC led to an impaired liver regeneration together with ATP depletion, induction of lipid peroxidation, alteration of protein synthesis, mitochondrial damage and apoptosis. The exact relationship between ROS production, mitochondrial damage and ER stress is not completely elucidated. It has been suggested that accumulation of unfolded proteins in the ER lumen triggers ROS production that in turn participates to induction of UPR and apoptosis. Misfolded proteins bind chaperones that consume ATP and may stimulate mitochondrial oxidative phosphorylation to generate ROS as a byproduct. They also lead to $\mathrm{Ca}^{2+}$ release from ER and uptake into mitochondria that disrupt the electron transport chain. ${ }^{22}$ Finally, disulfide bond formation in ER during the transfer of electrons from thiol groups to folding substrates also produces ROS. ${ }^{23}$ Here we demonstrate, for the first time, that liver resection alone or associated with intermittent pedicular clamping modulates ER stress and UPR. Particularly, we show that both kinetics and amplitude of UPR vary with surgical procedures and correlate with lipid peroxidation and ATP levels. A transient UPR activation and MDA level reduction were observed in the $\mathrm{RPH}$ group as compared with the TCPH group. In accordance, the ATP stores were better preserved in RPH than in TCPH group. Although both chaperones (GRP78 and GRP94), known to be specifically modulated by the ER stress, ${ }^{15}$ were detected $3 \mathrm{~h}$ after hepatectomy in the $\mathrm{PH}, \mathrm{NCPH}$ and $\mathrm{TCPH}$ groups, their mRNA and protein levels were highly increased in the RPH group. Numerous reports indicate that chaperone induction 


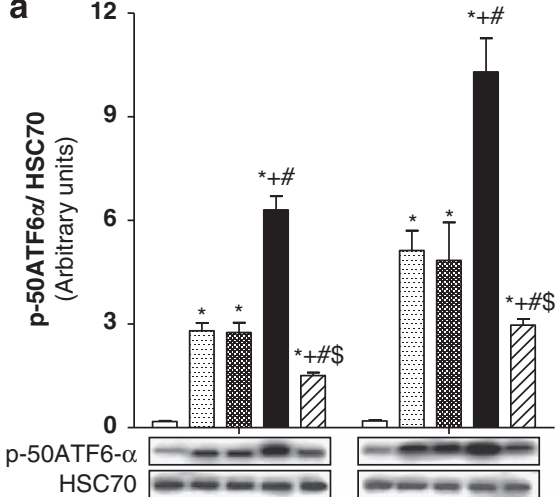

$3 \mathrm{~h}$

$24 \mathrm{~h}$

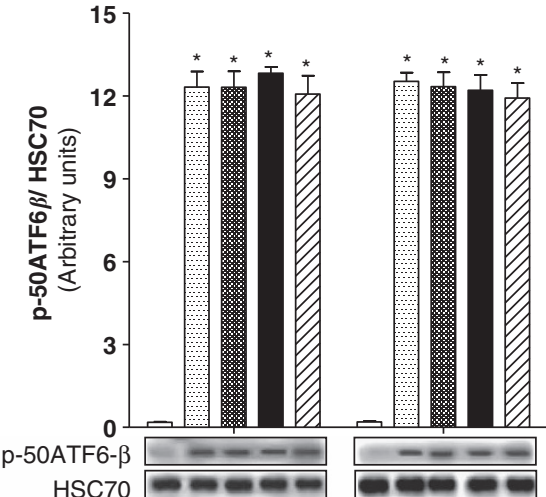

3h

$24 \mathrm{~h}$
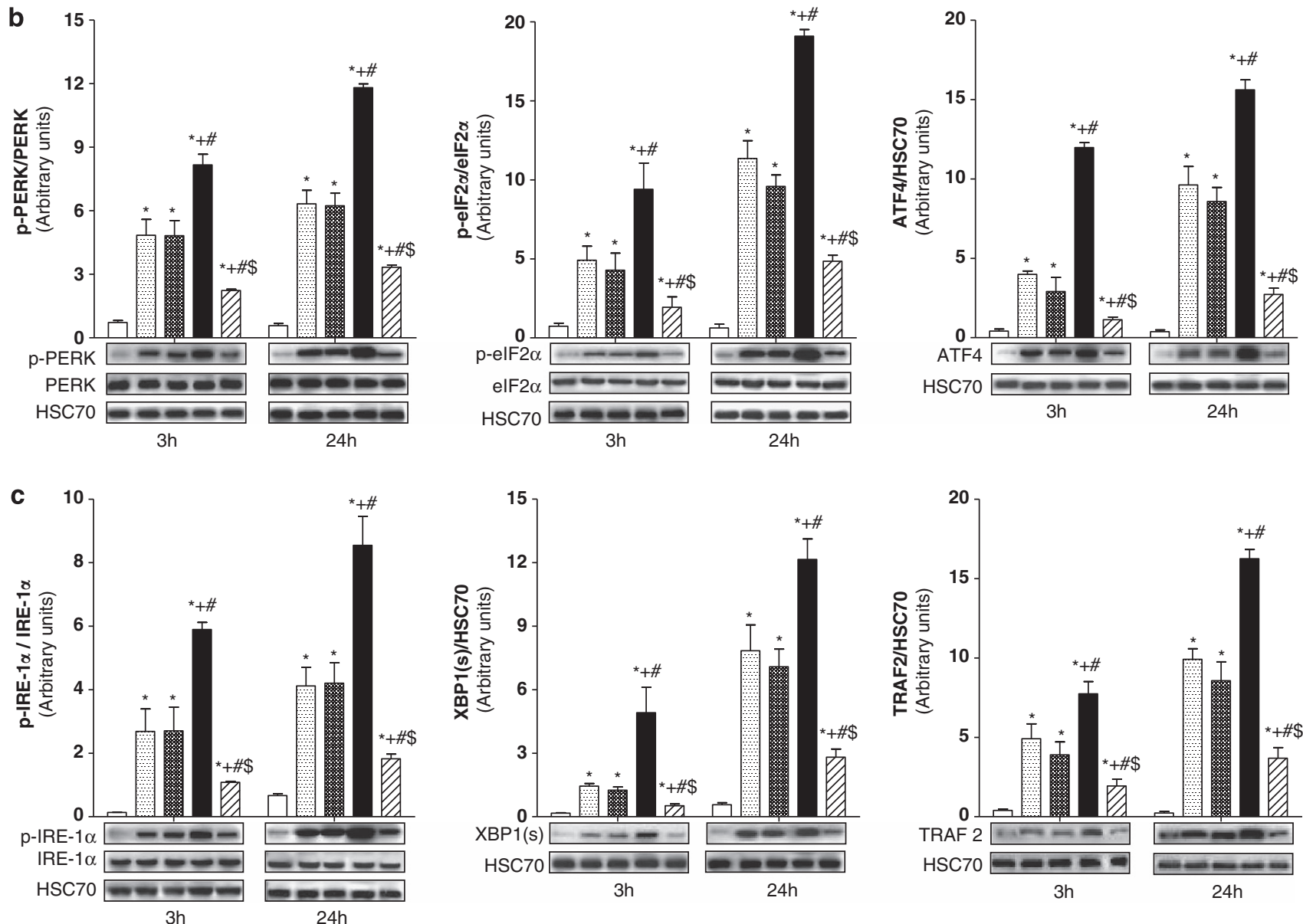

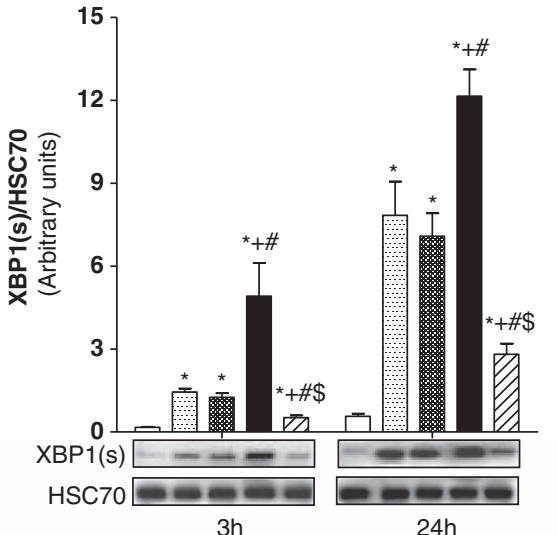

$3 h$

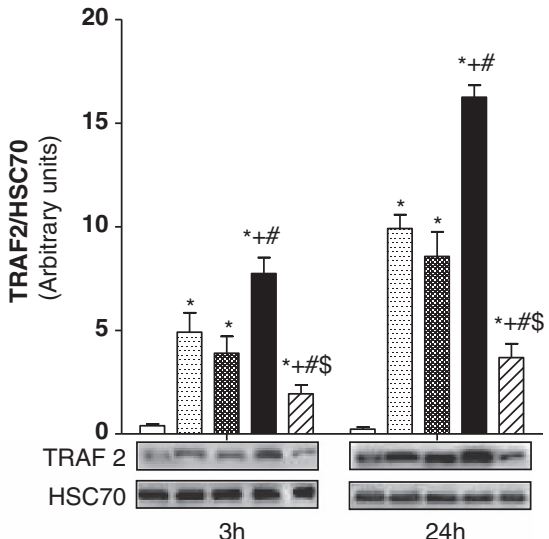

SHAM $\mathrm{PH}$ NCPH

$\mathrm{TCPH}$

D RPH

Figure 5 ISC limits ATF6, PERK and IRE- $1 \alpha$ pathway activation. p-50ATF6 $\alpha$ and p-50ATF6 $\beta$ (a), p-PERK, p-elF2 $\alpha$ and ATF4 (b), and p-IER-1 $\alpha$, XBP-1(S) and TRAF2 (c) expressions were detected by western blot 3 and 24 h post hepatectomy. ${ }^{+} P<0.05$ versus $\mathrm{PH},{ }^{\#} P<0.05$ versus NCPH and ${ }^{\$} P<0.05$ versus TCPH (N=5)

plays a key role in maintaining viability of cells subjected to stress by supporting proper folding and secretion of proteins. ${ }^{15,17}$ In agreement, a rapid chaperone induction in $\mathrm{RPH}$ group led to reduced ER stress intensity at $24 \mathrm{~h}$ post hepatectomy compared with the $\mathrm{PH}, \mathrm{NCPH}$ and $\mathrm{TCPH}$ groups, as evidenced by lower mRNA and protein levels of chaperones and $\mathrm{CHOP}$, the latter being a reliable indicator for ER stress-induced apoptosis. ${ }^{15}$ Moreover, transient activation of chaperones was associated with reduced activation of the three URP sensors, PERK, IRE1 and ATF6 $\alpha$, and their downstream target genes (eilF2 $\alpha$, ATF4, XBP-1(S), TRAF2, ASNS, EDEM and GADD34) at 3 and $24 \mathrm{~h}$ post hepatectomy. Among ATF6 isoforms, only ATF6 $\alpha$ is involved in resistance to ER stress. ${ }^{24}$ It is directly linked to the quality control of protein folding, involving the degradation of misfolded proteins and the restoration of the correct protein folding. ${ }^{15,17}$ Here, the 


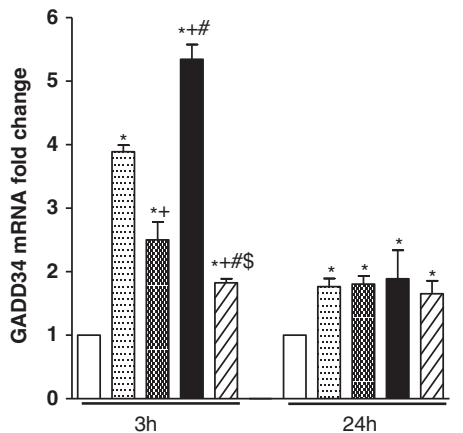

b

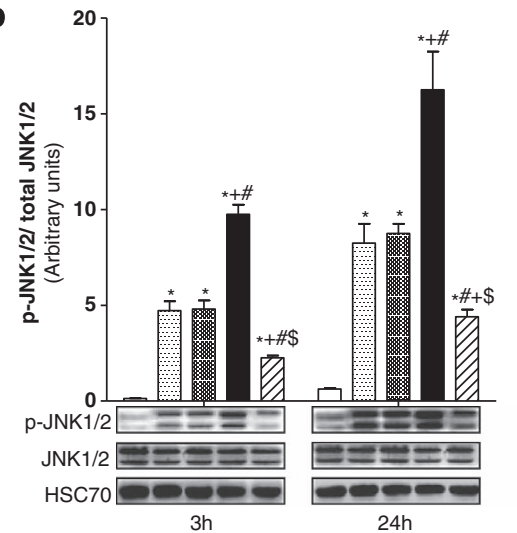

C

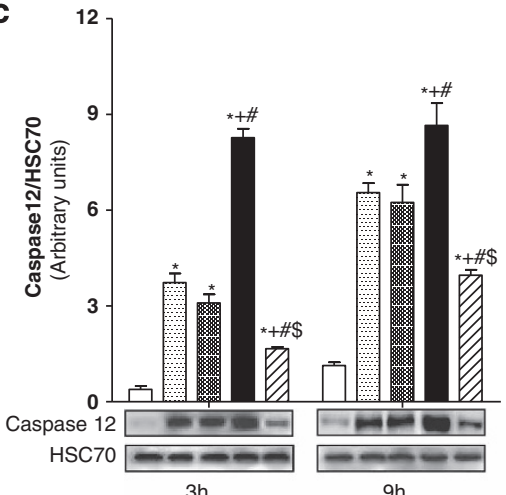

d

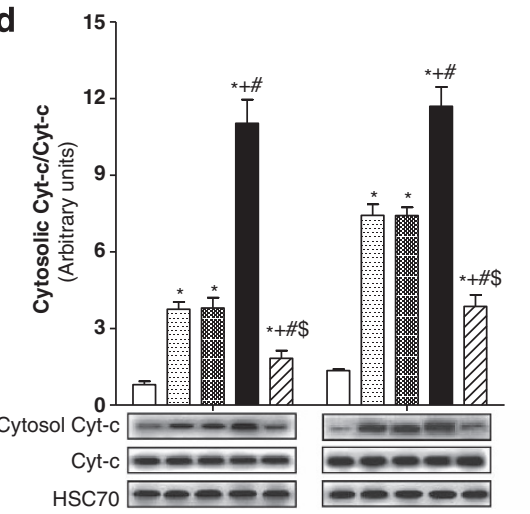

$3 \mathrm{~h}$
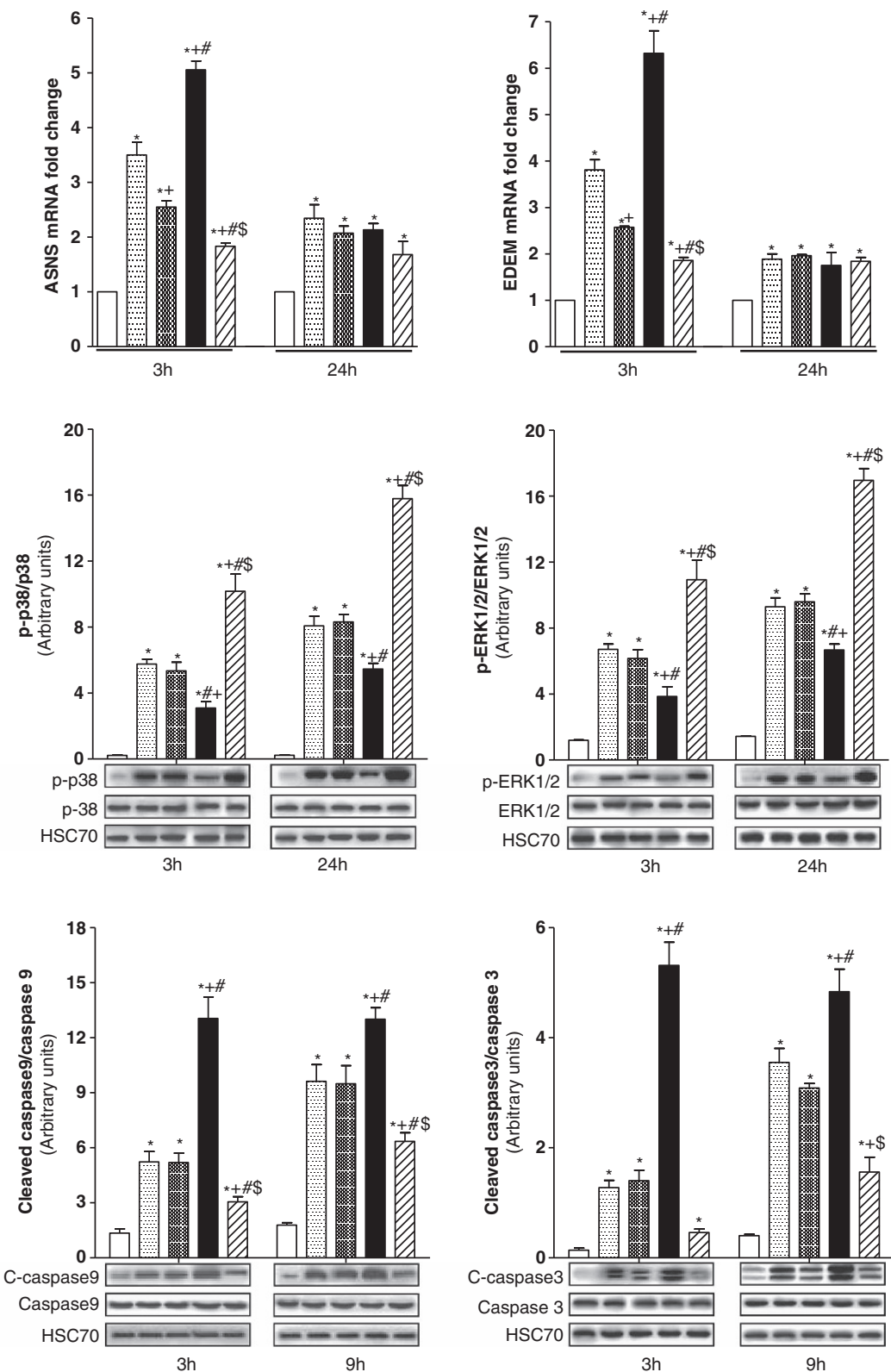

e
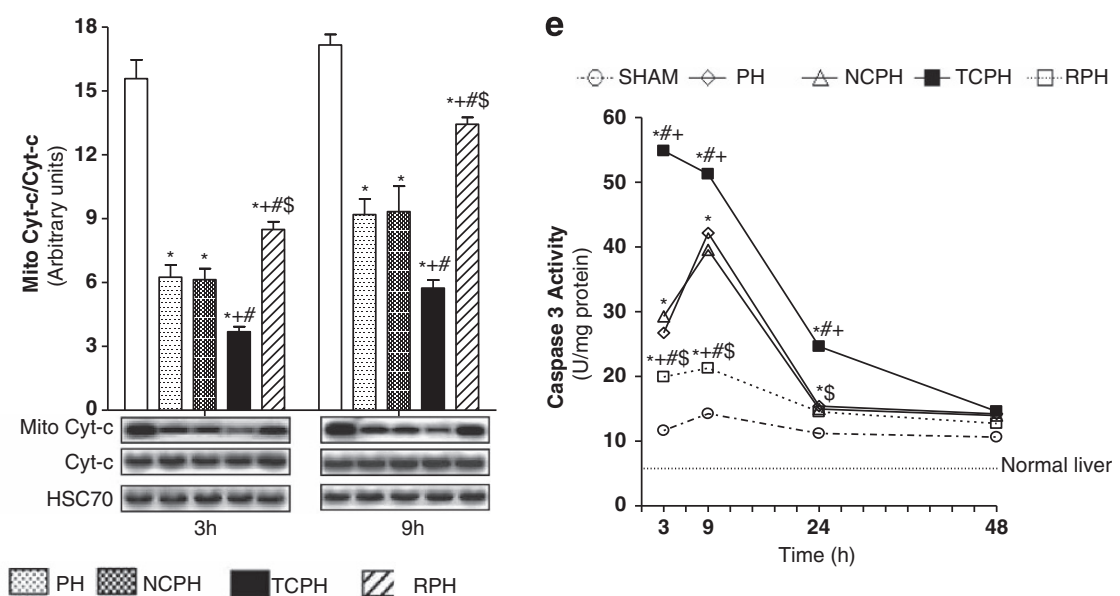
slight induction of ATF6 $\alpha$, PERK and IRE-1 was associated with the maintenance of albumin and transferrin production demonstrating a better preserved protein folding capacity in the RPH group.

It has been proposed that IRE-1 pathway regulates proapoptotic responses by activating the stress kinase JNK1/2, ${ }^{17}$ mitogen-activated protein kinase ERK1/2 and p38. ${ }^{25}$ However, these kinases play dual roles as they also promote cell survival and liver regeneration. ${ }^{26,27}$ Under our experimental conditions, lower activation of IRE-1 and of its downstream targets (XBP-1(S), TRAF2 and p-JNK1/2) were observed in the RPH group as compared with other groups, especially with the TCPH group. Conversely, important phosphorylation of p38 and ERK1/2 was observed in the RPH group. As apoptosis signaling cascades were barely activated in the $\mathrm{RPH}$ group as compared with other groups, we assume that both MAPKs activated survival and proliferation pathways after hepatectomy. We previously evidenced that the rapid initiation of liver regeneration was associated with a transient activation of JNK pathway just before liver resection in both $\mathrm{RPH}$ and NCPH groups. ${ }^{19}$ In the present study, IRE- $1 \alpha$ was strongly increased 3 and $24 \mathrm{~h}$ post hepatectomy in the TCPH group leading to sustained JNK and reduced MAPK activation. These events correlated with amplified caspase 3 activity and impaired liver regeneration. Having in mind that activated IRE- $1 \alpha$ binds TRAF2 that promotes p38 and ERK1/2 activation, our results suggest that other signaling mechanisms are involved in the damaging or protective effect induced by intermittent total or selective clamping, respectively. The balance between JNK and p38 and ERK1/2 activation intensity and time course plays a critical role in cell surviving and liver regeneration.

Endothelial NOS plays a key role in the immediate response to increased shear stress following hepatectomy. ${ }^{28}$ Thereby, low concentration of NO produced by eNOS serves to mitigate the hepatic microcirculation alterations and thus promotes cell survival. $^{28}$ Improved microcirculation and endothelial cell preservation increase availability of oxygen to hepatocyte and, therefore, facilitate ATP production. Shear stressinduced eNOS activation and NO release are also important mediators of hepatocyte proliferation after hepatectomy as ERK signaling was shown to be dependent of eNOS expression in regenerating livers. ${ }^{28}$ Our results demonstrate that eNOS pathways participate in the beneficial effects of ISC. Indeed, in RPH, increased eNOS expression, NO production, ATP stores and activation of ERK signaling were observed. This NO production derived mainly from eNOS activation as iNOS was barely expressed. Interestingly, it was suggested that the presence of $\mathrm{NO}$ increased GRP78 expression and regulated the flux of $\mathrm{Ca}^{2+}$ between the mitochondria, the Golgi and the ER to confer organ protection. Therefore, we can hypothesize that a positive ER environment induced by ISC favors hepatocyte survival and proliferation. In contrast, endothelial cell dysfunction,
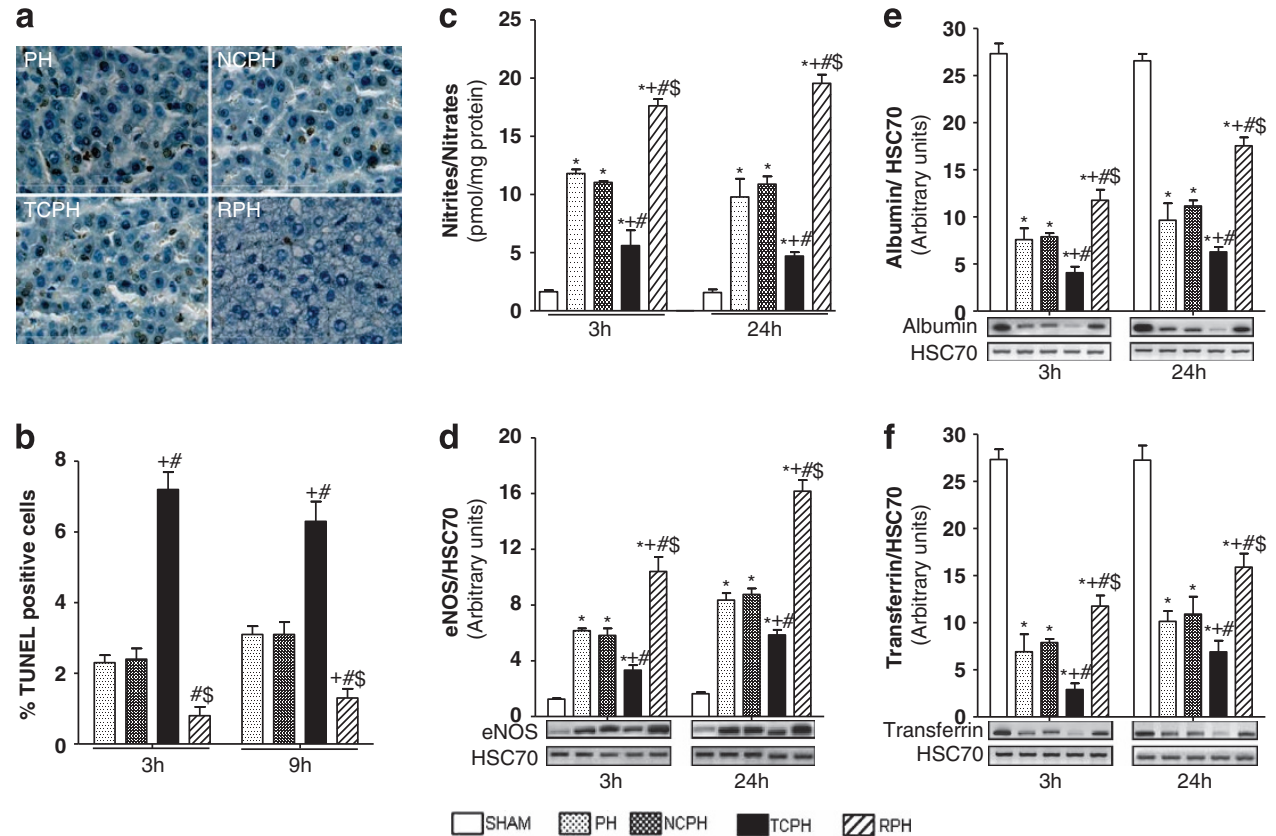

Figure 7 ISC reduces apoptosis and improves liver function. Immunohistochemical detection of TUNEL-positive cells (a) was analyzed $9 \mathrm{~h}$ post hepatectomy $(\times 40)$. (b) Percentage of TUNEL-positive hepatocytes was determined 3 and $9 \mathrm{~h}$ post hepatectomy. Nitrite/nitrate levels (c) and eNOS (d), albumin (e) and transferrin (f) expressions were evaluated by western blot 3 and $24 \mathrm{~h}$ post hepatectomy. ${ }^{*} P<0.05$ versus SHAM, ${ }^{+} P<0.05$ versus $\mathrm{PH},{ }^{\#} P<0.05$ versus $\mathrm{NCPH}$ and ${ }^{\$} P<0.05$ versus TCPH $(N=5)$

Figure 6 ISC attenuates the execution of ER stress and induces survival signaling through MAPK activation. GADD34, ASNS and EDEM mRNA expressions (a) were analyzed 3 and $24 \mathrm{~h}$ post hepatectomy. Phosphorylated-JNK1/2, p38 and ERK1/2 (b), caspase 12, cleaved caspases 9 and 3 (c) and cytosolic or mitochondrial cytochrome $c$ (d) expressions were evaluated by western blot at the indicated times. Caspase 3 activity (e) was determined at the indicated times. ${ }^{*} P<0.05$ versus SHAM, ${ }^{+} P<0.05$ versus $\mathrm{PH},{ }^{\#} P<0.05$ versus $\mathrm{NCPH}$ and ${ }^{\$} P<0.05$ versus $\mathrm{TCPH}(N=5)$ 
imbalance in ROS, NO and ATP production, reduced antioxidant activities and prolonged UPR activation result in increased apoptosis and delayed liver regeneration.

Taken together, our data demonstrate for the first time that adaptation of ER to liver resection stress plays an important role in efficient liver regeneration response. They also show that UPR signaling is a central target through which short intermittent reperfusion stress exerts its protective effect and improves liver regeneration. In light of these results, the commonly used total clamping could be revisited in favor of an ISC when applicable to stimulate regeneration, and reduce hepatic insufficiency. This procedure could be applied as a powerful protective modality in the field of living donor liver transplantation and liver surgery.

\begin{abstract}
Materials and Methods
Animals and surgical procedures. All experiments were conducted according to the European Union regulations (Directive 86/609 EEC) for animal experiments and complied with our institution's guidelines for animal care and handling (permit number 35-16 delivered to Dr. Anne Corlu, 08/06/2010). Male Sprague-Dawley rats weighing 220 to $280 \mathrm{~g}$ (Janvier, Le Genest-St-Isle, France) were kept on a standard diet and water ad libitum. For each time point $(3,9,18$, $21,24,30$ and 48 h), 25 rats were divided equally and randomly allocated into five groups (Figure 1). In SHAM group, left lateral and median lobes that represent $70 \%$ of the liver were exteriorised for $30 \mathrm{~min}$ and mobilized at the indicated times to simulate the clamping and unclamping procedures. No hepatectomy was performed. In standard hepatectomy group ( $\mathrm{PH})$, the left lateral and median lobes were immediately excised according to the procedure described by Higgins and Anderson ${ }^{29}$ without any prior mobilization. In nonclamping group (NCPH), rats underwent the same protocol as SHAM followed by left lateral and median lobe resection. In total clamping group (TCPH), occlusion of the entire hepatic pedicle was performed using micro-bulldog clamps and livers were subjected twice to $10 \mathrm{~min}$ of ischemia followed by $5 \mathrm{~min}$ of reperfusion before partial hepatectomy. Finally, in reperfusion group (RPH), only lobes to be resected were exteriorised and subjected twice to $10 \mathrm{~min}$ of ischemia followed by $5 \mathrm{~min}$ of reperfusion before hepatectomy (Figure 1). At different time points after hepatectomy $(3,9,18,21$, 24,30 and $48 \mathrm{~h}$ ), animals were killed under pentobarbital anesthesia. Blood was collected. Livers were snap-frozen in liquid nitrogen and stored at $-80^{\circ} \mathrm{C}$ or fixed in $4 \%$ formaldehyde.
\end{abstract}

Biochemical determinations. ALT, AST, LDH, MDA, ATP, nitrite/nitrate levels and catalase, SOD and caspase 3 activities were measured as described elsewhere. ${ }^{13,30}$ Results were expressed as mean \pm S.D. of five rats per group.

DNA synthesis and mitotic index. Percentage of proliferating hepatocytes was estimated by the quantitation of hepatocytes that incorporated BrdU antigen. ${ }^{19,31}$ At least an average of 4000 hepatocytes/liver section was scored in adjacent fields using an Olympus microscope and simple $\mathrm{PCl}$ software (ImPACcell platform, Rennes, France). The mitotic index was determined using Hoechst staining. ${ }^{19,31}$ Positive cells were evaluated in a blind manner by two different investigators who counted at least 10 random high-power fields on each section.

Western blot analysis. Liver tissues were homogenized and subcellular fractions were resolved on $12 \%$ sodium dodecyl sulfate (SDS)-PAGE, transferred onto nitrocellulose membrane and analyzed as previously described using chemiluminescence detection. ${ }^{13,32}$ Total liver lysates were used to quantify PCNA, GRP78, GRP94, CHOP, ATF4, p50-ATF6 $\alpha$, p50-ATF6 $\beta$, XBP-1(S), TRAF2, HSC70, caspase 12, cytochrome $c$, cleaved caspase 3 , caspase 9 , eNOS and iNOS, albumin, transferrin, total and phospho-PERK, -IRE-1, -elF2 $\alpha,-p 38$ and -JNK1/2. Cytosolic and mitochondrial fractions were used to quantify cleaved caspase 9 and/or cytochrome $c .13,30$ Proteins were separated by SDS-PAGE and transferred to polyvinylidene fluoride (PVDF) membranes. Membranes were immunoblotted with antibodies directed against PCNA (Dako, Les Ulis, France) GRP78, ATF6 $\alpha$, ATF6 $\beta$, GRP94, CHOP, ATF4, XBP-1(S), Caspase 12, total and phospho-PERK, total and phospho-elF2 $\alpha$ (Santa Cruz Biotechnology, Santa Cruz,
CA, USA), TRAF2, cytochrome $c$, cleaved caspase 3 , cleaved caspase 9 , total and phospho-JNK1/2, total and phospho-p38 MAPK (Cell Signaling Technology Inc., Beverly, MA, USA), total and phospho-IRE-1 (Thermo Fisher Scientific, Illkirch, France), eNOS and iNOS (Transduction Laboratories, Lexington, KY, USA), albumin and transferrin (Bethyl Laboratories, Inc., Euromedex, Souffelweyersheim, France). Protein expression was visualized using an enhanced chemiluminescence kit (ECF; Amersham Biosciences, Orsay, France) and signals were quantified with scanning densitometry using the Quantity One software program (Bio-Rad Laboratories, Hercules, CA, USA). Representative western blots represent one of three independent experiments with similar results. All signals were normalized with the loading control HSC70, for PCNA, GRP78, GRP94, CHOP, ATF4, p-50ATF6 $\alpha$, XBP-1(S), TRAF2, caspase 12, eNOS, albumin and transferrin and with total PERK, elF2 $\alpha$, IER-1 $\alpha$, JNK1/2, p38, ERK1/ 2 , caspases 3 and 9 and cytochrome $c$ for p-PERK, p-eIF2 $\alpha$, p-IER- $1 \alpha, p$-JNK1/2, $p-p 38, p-E R K 1 / 2$, cleaved caspases 3 and 9 and (cytosolic and mitochondrial), respectively. All relative expressions were compared with those of normal liver (NL) arbitrary set at one.

Reverse transcription and RT-PCR. Total RNA was extracted using Tri-reagent (Invitrogen, Saint Aubin, France) according to the manufacturer's instructions and treated with DNase I. Then, $1 \mu \mathrm{g}$ of total RNA was reversetranscribed using M-MLV reverse transcriptase and random primers (Invitrogen). The TaqMan gene expression assay was performed according to the manufacturer's protocol (Applied Biosystems, Foster City, CA, USA) using the Assays-on-Demand TaqMan probe (Rn00565250 for GRP78, Rn01760569 for GRP94, Rn00492098 for CHOP, Rn00591894 for GADD34, Rn01421307 for EDEM and Rn00565180 for ASNS). Relative quantification of the steady-state target mRNA levels was calculated after normalization of the total amount of CDNA tested to the 18S, and mRNA expressions in liver from SHAM were arbitrary set at one.

Histology, immunohistology and TUNEL assay. For standard histology evaluation, hematoxylin/eosin staining was performed and the morphological liver integrity was graded on a scale as previously described. ${ }^{30,33}$ Immunohistological studies were performed to evaluate GRP78- and CHOPpositive cells. Apoptosis was assessed by TUNEL as described elsewhere. ${ }^{30}$ Damage score, ratio of GRP78- and CHOP-positive cells as well as TUNELpositive nuclei were evaluated in a blind manner by two different investigators who counted at least 10 random high-power fields on each section.

Statistical analysis. Data were expressed as means \pm S.D. Statistical analysis was performed by variance analysis (Kruskal-Wallis test), followed by Mann-Whitney $U$ test. $P<0.05$ was considered as significant.

\section{Conflict of Interest}

The authors declare no conflict of interest.

Acknowledgements. We thank Dr. Bruno Turlin (CHU Rennes) and Mrs. Pascale Bellaud (Histo-pathological platform, Rennes, UMS biosit, Biogenouest) for histological analysis and Dr. Rémy Le Guevel for image analyses (ImPACcell platform, Rennes, UMS Biosit, Biogenouest). We thank Dr. Pascal Loyer for helpful comments and critical reading of the article. This work was supported by the Institut National de la Santé et de la Recherche Médicale, the Centre National de la Recherche Scientifique, the Fonds Européen de Développement Régional (FEDER) and the 'Contrat projets état région' (axe biothérapie). IBM was funded by the 'Contrat plan état région' (axe biothérapie) and the 'Fondation pour la Recherche Médicale'. JP was funded by the 'Conseil Régional de Bretagne' and the 'Ligue Contre le Cancer- Comité d'llle-et-Vilaine'. The funders had no role in study design, data collection and analysis, decision to publish or preparation of the manuscript.

1. Man K, Fan ST, Ng IO, Lo CM, Liu CL, Wong J. Prospective evaluation of Pringle maneuver in hepatectomy for liver tumors by a randomized study. Ann Surg 1997; 226: 704-711; discussion 711-703.

2. Belghiti J, Noun R, Malafosse R, Jagot $P$, Sauvanet A, Pierangeli $F$ et al. Continuous versus intermittent portal triad clamping for liver resection: a controlled study. Ann Surg 1999; 229: 369-375. 
3. Wei AC, Tung-Ping Poon R, Fan ST, Wong J. Risk factors for perioperative morbidity and mortality after extended hepatectomy for hepatocellular carcinoma. Br J Surg 2003; 90 33-41.

4. Rudiger HA, Kang KJ, Sindram D, Riehle HM, Clavien PA. Comparison of ischemic preconditioning and intermittent and continuous inflow occlusion in the murine liver Ann Surg 2002; 235: 400-407.

5. Malhi H, Kaufman RJ. Endoplasmic reticulum stress in liver disease. J Hepatol 2011; 54: 795-809.

6. Chang WJ, Chehab M, Kink S, Toledo-Pereyra LH. Intracellular calcium signaling pathways during liver ischemia and reperfusion. J Invest Surg 2010; 23: 228-238.

7. Glantzounis GK, Salacinski HJ, Yang W, Davidson BR, Seifalian AM. The contemporary role of antioxidant therapy in attenuating liver ischemia-reperfusion injury: a review. Liver Transpl 2005; 11: 1031-1047.

8. Kaplowitz N, Than TA, Shinohara M, Ji C. Endoplasmic reticulum stress and liver injury. Semin Liver Dis 2007; 27: 367-377.

9. Walter $P$, Ron $D$. The unfolded protein response: from stress pathway to homeostatic regulation. Science 2011; 334: 1081-1086.

10. Zhang K, Kaufman RJ. From endoplasmic-reticulum stress to the inflammatory response. Nature 2008; 454: 455-462.

11. Zhang C, Wang G, Zheng Z, Maddipati KR, Zhang X, Dyson G et al. Endoplasmic reticulum-tethered transcription factor CAMP responsive element-binding protein, hepatocyte specific, regulates hepatic lipogenesis, fatty acid oxidation, and lipolysis upon metabolic stress in mice. Hepatology 2012; 55: 1070-1082.

12. Peralta $C$, Brenner $C$. Endoplasmic reticulum stress inhibition enhances liver tolerance to ischemia/reperfusion. Curr Med Chem 2011; 18: 2016-2024.

13. Ben Mosbah I, Alfany-Fernandez I, Martel C, Zaouali MA, Bintanel-Morcillo M, Rimola A et al. Endoplasmic reticulum stress inhibition protects steatotic and non-steatotic livers in partial hepatectomy under ischemia-reperfusion. Cell Death Dis 2010; 1: e52.

14. Malhi $\mathrm{H}$, Gores GJ. Cellular and molecular mechanisms of liver injury. Gastroenterology 2008; 134: 1641-1654

15. Zhang K, Kaufman RJ. Identification and characterization of endoplasmic reticulum stress-induced apoptosis in vivo. Methods Enzymol 2008; 442: 395-419.

16. Ozcan U, Yilmaz E, Ozcan L, Furuhashi M, Vaillancourt E, Smith RO et al. Chemica chaperones reduce ER stress and restore glucose homeostasis in a mouse model of type 2 diabetes. Science 2006; 313: 1137-1140.

17. Urano F, Wang X, Bertolotti A, Zhang Y, Chung P, Harding HP et al. Coupling of stress in the ER to activation of JNK protein kinases by transmembrane protein kinase IRE1. Science 2000; 287: 664-666.

18. Xu C, Bailly-Maitre B, Reed JC. Endoplasmic reticulum stress: cell life and death decisions. J Clin Invest 2005; 115: 2656-2664.

19. Duval H, Mbatchi SF, Grandadam S, Legendre C, Loyer P, Ribault C et al. Reperfusion stress induced during intermittent selective clamping accelerates rat liver regeneration through JNK pathway. J Hepatol 2010; 52: 560-569.

20. Weigand K, Brost S, Steinebrunner N, Buchler M, Schemmer P, Muller M. Ischemia/ reperfusion injury in liver surgery and transplantation: pathophysiology. HPB Surg 2012; 2012: 176723.
21. Giakoustidis DE, Iliadis S, Tsantilas D, Papageorgiou G, Kontos N, Kostopoulou E et al. Blockade of Kupffer cells by gadolinium chloride reduces lipid peroxidation and protects liver from ischemia/reperfusion injury. Hepatogastroenterology 2003; 50: 1587-1592.

22. Deniaud A, Sharaf el dein O, Maillier E, Poncet D, Kroemer G, Lemaire C et al. Endoplasmic reticulum stress induces calcium-dependent permeability transition, mitochondrial outer membrane permeabilization and apoptosis. Oncogene 2008; 27 : 285-299.

23. Chai $F$, Luo R, Li Y, Bai Y, He Y, Wei Y et al. Down-regulation of GRP78 in human glaucomatous trabecular meshwork cells. Mol Vis 2010; 16: 1122-1131.

24. Yamamoto K, Sato T, Matsui T, Sato M, Okada T, Yoshida H et al. Transcriptional induction of mammalian ER quality control proteins is mediated by single or combined action of ATF6alpha and XBP-1 (S). Dev Cell 2007; 13: 365-376.

25. Nguyen DT, Kebache S, Fazel A, Wong HN, Jenna S, Emadali A et al. Nck-dependent activation of extracellular signal-regulated kinase-1 and regulation of cell survival during endoplasmic reticulum stress. Mol Biol Cell 2004; 15: 4248-4260.

26. Ramalho FS, Alfany-Fernandez I, Casillas-Ramirez A, Massip-Salcedo M, Serafin A Rimola $A$ et al. Are angiotensin II receptor antagonists useful strategies in steatotic and nonsteatotic livers in conditions of partial hepatectomy under ischemia-reperfusion? J Pharmacol Exp Ther 2009; 329: 130-140.

27. Campbell JS, Argast GM, Yuen SY, Hayes B, Fausto N. Inactivation of p38 MAPK during liver regeneration. Int J Biochem Cell Biol 2011; 43: 180-188.

28. Mei $Y$, Thevananther $S$. Endothelial nitric oxide synthase is a key mediator of hepatocyte proliferation in response to partial hepatectomy in mice. Hepatology 2011; 54: 1777-1789.

29. Higgins GM, Anderson RM. Experimental pathology of the liver. I: restoration of the liver of the white rat following partial surgical removal. Arch Pathol 1931; 12: 186-202.

30. Ben Mosbah I, Mouchel Y, Pajaud J, Ribault C, Lucas C, Laurent A et al. Pretreatment with mangafodipir improves liver graft tolerance to ischemia/reperfusion injury in rat. PLoS One 2012; 7: e50235.

31. Furrer K, Deoliveira ML, Graf R, Clavien PA. Improving outcome in patients undergoing liver surgery. Liver Int 2007; 27: 26-39.

32. Puri $\mathrm{P}$, Mirshahi $\mathrm{F}$, Cheung $\mathrm{O}$, Natarajan R, Maher JW, Kellum JM et al. Activation and dysregulation of the unfolded protein response in nonalcoholic fatty liver disease. Gastroenterology 2008; 134: 568-576.

33. $t$ Hart NA, van der Plaats A, Leuvenink HG, Wiersema-Buist J, Olinga $P$, van Luyn MJ et al. Initial blood washout during organ procurement determines liver injury and function after preservation and reperfusion. Am J Transplant 2004; 4: 1836-1844.

(c) (i) () $\Theta$ Cell Death and Disease is an open-access journal published by Nature Publishing Group. This work is licensed under a Creative Commons Attribution-NonCommercialNoDerivs 3.0 Unported License. To view a copy of this license, visit http://creativecommons.org/licenses/by-nc-nd/3.0/ 